\title{
A Phase-based Method for Mobile Node Localization through UHF-RFID passive tags
}

\author{
Andrea Motroni, Paolo Nepa \\ Dept. Information Engineering \\ University of Pisa \\ Pisa, Italy \\ andrea.motroni@ing.unipi.it,paolo.nepa@unipi.it
}

\author{
Alice Buffi, Bernardo Tellini \\ Dept. Energy, Systems, Territory and Costructions Engineering \\ University of Pisa \\ Pisa, Italy \\ alice.buffi@unipi.it, bernardo.tellini@unipi.it
}

\begin{abstract}
This paper presents a novel phase-based localization method for mobile nodes equipped with a UHF-RFID system. The on-board reader collects phase data from a set of passive reference tags during the mobile node trajectory, by forming a synthetic array. This allows the adoption of a low-density distribution of reference tags. Then, the phase data are combined with information acquired by low-cost kinematic sensors through a Sensor Fusion approach. The method can be employed in both indoor and outdoor scenarios and independently on the reference tag positions. The proposed system represents a low-cost and easy deployable solution to reduce the typical drift of dead-reckoning algorithms for localization.
\end{abstract}

Keywords-Mobile Node Localization, UHF-RFID Mobile Node, UHF-RFID Sensor Fusion, UHF-RFID Localization.

\section{INTRODUCTION}

The localization issue of mobile nodes, such as robots, vehicles or humans, has reached great attention in recent years [1]. The knowledge of the node position is useful in several applications such as environmental monitoring, access control, target tracking, intrusion detection and so on.

In outdoor scenario, typical solutions exploit satellite navigation systems (e.g. GPS, GLONASS, GALILEO) which allow for localization errors in the order of few meters. However, they suffer from the presence of obstacles as for example the tree crowns. In indoor environments, different methods were proposed for mobile nodes positioning [2]. The simplest solutions for wheeled vehicles include the use of odometers through dead-reckoning algorithms. However, the encoder measurements accumulate error during the vehicle path by leading to a drift in position estimation [3]. To overcome this limitation, odometers are typically employed together with other sensors, such as sonar [4] or laser [5]. Sonars are cheap, but their performance is strictly dependent on the presence of obstacles and on the speed of the mobile node. Lasers allow to measure the distance of obstacles with centimetre order error and to create a 2D or 3D map of the scenario, despite of high cost. Alternatively, optical sensors (cameras) can be employed [6] together with sophisticated methods of image processing, even if they require a high computational cost. Besides, performance is strongly dependent on the scenario and the lighting conditions.

In this context, a radio-localization system based on RFID technology offers many advantages, allowing for a low-cost solution which does not require the optical visibility [7]-[8]. The first solutions exploited the RFID system at the HF frequency band (i.e. 13.56 MHz) [9]. The mobile node equipped with the HF-RFID reader detects the HF reference tags deployed at the floor side. The robot position is estimated as the position of the detected reference tag, thus the localization precision depends on the density of the reference tags themselves.

More recently the widespread solutions suggest the employment of the RFID systems at the UHF band. The mobile node can be equipped with a UHF-RFID tag and fixed reader antennas were employed to locate it [10], despite of high infrastructure cost. Alternatively, the mobile node can be equipped with the UHF-RFID reader and the tags are displaced at known positions in the scenario to perform positioning [11].

In this paper, a novel phase-based method for mobile node self-localization based on UHF-RFID passive technology is proposed. The mobile node equipped with the UHF-RFID reader collects phase data from reference tags in the scenario along its trajectory, resembling a synthetic array. This allows to reduce the number of required reference tags, since many readings from a single tag are available. Then, the phase samples are combined with the data collected by low-cost kinematic sensors (e.g. acceleration, angular speed) through a Sensor Fusion approach. The method can be applied independently on the reference tag positions which can be placed on the floor, on the ceiling or attached to fixed objects.

The paper is organized as follow. Section II presents the state-of-the-art on mobile node localization based on UHF-RFID system. Section III described the novel phase-based method for mobile node self-localization and the results of numerical analysis are in Section IV. Finally, the conclusion is drawn in Section V.

\section{UHF-RFID MOBILE NODE LOCALIZATION}

Several works in the state-of-the-art address the mobile node positioning issue through RFID systems at the UHF band. Typically, beyond the tag detection data [12], the amplitude of the tag backscattered signal can be employed (Received Signal Strength Indicator, RSSI) [13]-[14]. In [12]-[14] the mobile node (e.g. a robot) is equipped with a RFID reader and fixed RFID reference tags are deployed in the environment, then sensor fusion techniques or estimation algorithms such as Kalman Filter, Particle Filter and their variations were adopted for positioning. However, performance of RSSI-based solutions depends on the number and typology of reference tags, and they require long calibration procedure which has to be repeated if some changes occur in the scenario.

Phase-based localization methods [15] typically allow for a better localization accuracy, and they are more robust to the multipath propagation phenomena with respect to the amplitude-based methods as described in [16]. Two main issues in phasebased UHF-RFID localization exist: the ambiguity due to the phase periodicity and the phase offset term which affects the phase 
measurement and which is unknown a priori [15]. In [17] a tag localization method was proposed which relies on a fixed infrastructure of reader antennas. The phase measurement ambiguity is solved by using the Extended Kalman Filter (EKF) and the Rauch-Tung-Striebel (RTS) smoother, where the dynamic state includes the position, the velocity and the phase offset term for each antenna. Such a solution allows a root mean square (RMS) of the localization error equal to 1-2 cm in an indoor scenario of $3 \mathrm{~m} \times 3 \mathrm{~m}$ by employing four reader antennas.

In [18], a Sensor-Fusion approach was proposed to locate a mobile node equipped with custom tag operating at $5.8 \mathrm{GHz}$. The accelerometer data measured by an inertial sensor are combined with the RSSI and the phase data. A millimetre order RMS error was achieved at a range of several meters.

In [19], the robot localization was achieved through a multi-hypothesis EKF which combines the data from odometry sensor and the phase of the signal backscattered by reference tags. The latter are custom tags placed at the ceiling. A calibration procedure for each reference tag is required to estimate the phase offset term. Such method was extended in [20] by employing both RSSI and phase data. The new algorithm showed higher robustness with respect to the errors on the knowledge of the reference tag positions. Moreover, the phase offset calibration procedure was avoided. Experimental results showed that a robot can be localized with an error of around $4 \mathrm{~cm}$ in a $4 \mathrm{~m} \times 3 \mathrm{~m}$ room by employing two reference tags in the whole area. Finally, the authors declared that the method can be employed in scenario of arbitrary sizes by deploying a reference tag every $2 \mathrm{~m}^{2}$.

At the best of the authors' knowledge, the phase-based UHF-RFID localization methods based on a Sensor-Fusion approach at the state-of-the-art, still required a set of reference tags with high density.

\section{The Novel Phase-Based Localization Method}

The authors of this paper recently conceived a novel phase-based method for self-localization of mobile node by employing UHF-RFID technology [21]. Fig. 1 represents a scheme of the proposed system. The mobile node is equipped with Commercial off-the-shelf (COTS) UHF-RFID reader and antenna, a set of kinematic sensors such as accelerometer, gyroscope and magnetometer, and the self-localization module. The latter is based on a Sensor Fusion approach which combines the phase data acquired from the reference tags placed in the surrounding scenario with the information related to the mobile node acceleration and angular speed.

By performing measurements during the relative motion of the node with respect to the reference tags, it is possible to collect several phase data to form a synthetic array. This is the main novelty of the proposed self-localization method which allows to reduce the number of required reference tags. The collected phase variation of the tag backscattered signal varies according to the distance variation between the reader antenna and the tag, thus represents a peculiar parameter to estimate the mobile node trajectory.

The self-localization method can be realized with firmware or software implementation. The estimated trajectory can be employed within the navigation system of the mobile node or it can be transmitted to an external server or personal computer through a Wi-Fi network.

The novel phase-based localization method is based on the definition of a geometric and kinematic model of the whole dynamic system. By considering the Inertial Measurement Unit (IMU) measurements, a set of hypothetical trajectories of the mobile node can be built for a selected time interval. Then, a set of hypothetical phase curves can be derived by the knowledge of the reference tag position.

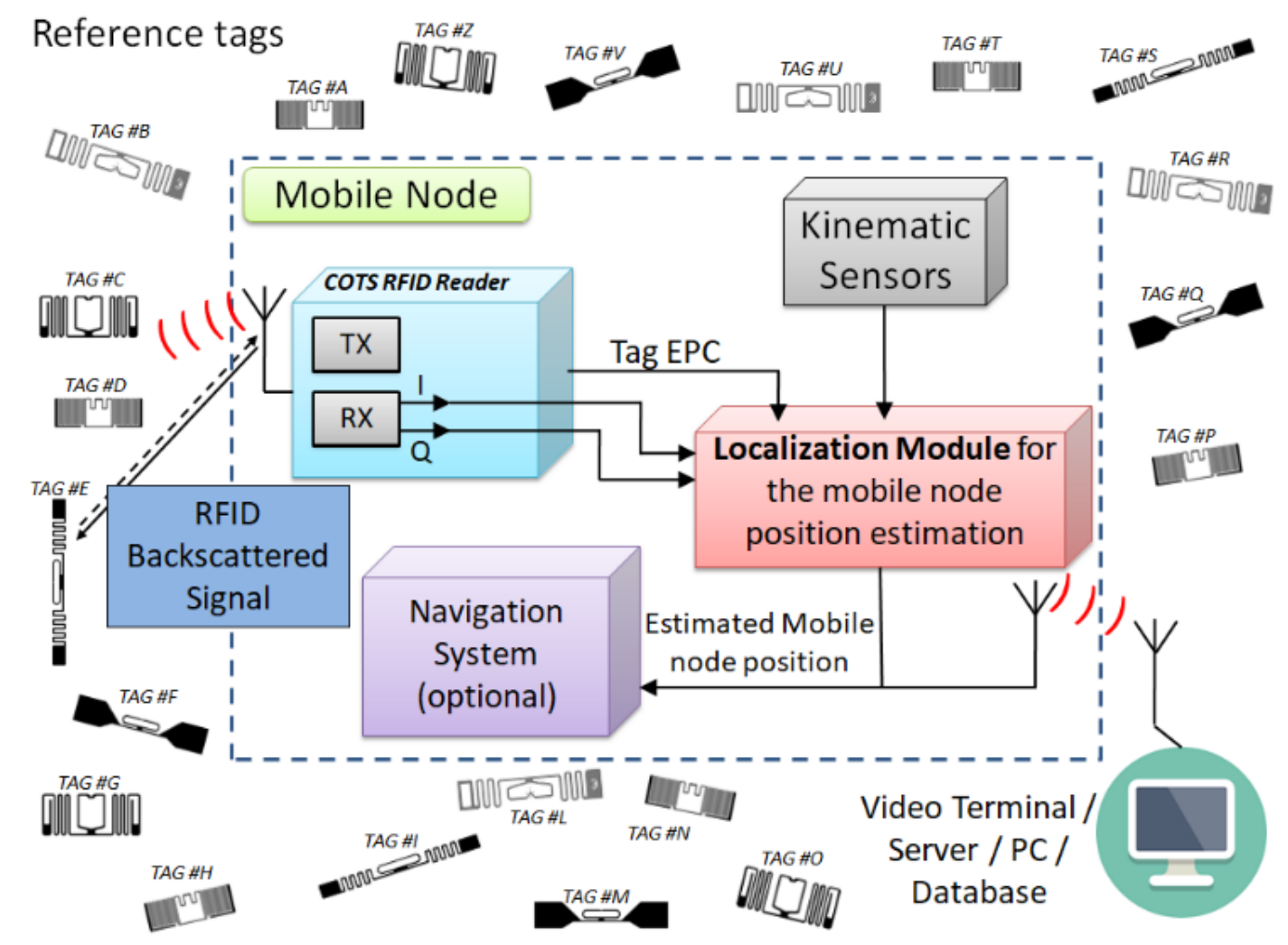


Fig. 1. Scheme of the mobile node equipped with UHF-RFID COTS hardware, kinematic sensors and the module implementing the novel UHF-RFID phase-based method for self-localization.

The latter are compared with the measured phase curve. The result of such comparison provides an output parameter, namely a weight, which is associated to the considered hypothetical trajectory. After that, according to a proper selection criterion, the hypothetical trajectories with the lower weight are eliminated. The mean value of the positions of the remaining hypothetical trajectories is adopted as the estimate of the trajectory portion run by the mobile node. The procedure is repeated as the mobile node moves, considering consecutive trajectory portions. Due to the elimination of hypothetical trajectories with low weight, the surviving hypothetical trajectories can be replicated after some iterations.

It is noteworthy that, the selection criteria based on the weight of the hypothetical trajectories represents a degree of freedom and it can be chosen on the basis of the particular application context. Similarly, the combination of the remaining hypothetical trajectories can be realized with several methods, such as a weighted average, maximum weight, and so on.

Finally, the reference tags can be placed in different locations of the indoor (e.g. ceiling, floor, pillars) or outdoor (e.g. lighting poles, road signs, trees) scenario, as long as the RFID reader detects at least one tag during each portion of its journey. They can be of different typologies and it is necessary to known their positions in a local reference system, at the time of the first installation. Expensive and time-consuming calibration procedures to preventively measure the tag backscattered signal are not required.

\section{NUMERICAL ANALYSIS}

The novel phase-based localization method was validated through a numerical analysis, by implementing a system simulator. Using the typical parameters of the commercial UHF-RFID readers and the characteristics of the RFID signal defined by international standards, EPC Global Class1 Gen 2, the simulator allows for the analysis of the performance when different parameters change: speed and trajectory of the mobile node, density and configuration of the reference tags, signal to noise ratio, level of interference caused by reflections from multiple paths (multipath) due to the presence of obstacles, uncertainty in the phase measurements from the reference tags, uncertainty in the measurement of acceleration and speed by kinematic sensors.

Without loss of generality, we consider a scenario in which the reference tags are arranged at a height greater than the plane in which the mobile node moves, as for example when they are attached at the ceiling. The RFID antenna placed on the mobile node is considered facing upwards. The set of kinematic sensors is composed by an IMU containing a 3-axis accelerometer and a 3-axis gyroscope.

Other parameters employed in the numerical analysis through the paper are as follow:

- $f_{0}=867.5 \mathrm{MHz}$, operating frequency (ETSI channel 4);

- $\Delta h=3 \mathrm{~m}$, difference between the heights of reference tags and reader antenna;

- $\Delta t \sim 10 \mathrm{~ms}$, average time difference between two consecutive tag readings (nearby 100 readings per second);

- $H P B W=65^{\circ}$, half-power beam width of the reader antenna;
- $\sigma_{a}=1.7 \cdot 10^{-3} \mathrm{~g}$, standard deviation of each component of mobile node acceleration;

- $\sigma_{\omega}=2.44 \cdot 10^{-3} \mathrm{rad} / \mathrm{s}$, standard deviation of each component of mobile node angular speed.

Generally speaking, the number of phase samples available for each tag depends on the reader query period, the antenna half power beam width, the distance between the antenna and the tag, and the speed of the mobile node.

We suppose that the mobile node moves along a " $\mathrm{S}$ shaped" trajectory (solid line in Fig. 2) with a speed of $1 \mathrm{~m} / \mathrm{s}$. Other system parameters are as follow: $a_{x} \in[-1,1] \mathrm{m} / \mathrm{s}^{2}$, $a_{y} \in[-1,1] \mathrm{m} / \mathrm{s}^{2}, a_{z}=0$, and $\omega=1 \mathrm{rad} / \mathrm{s}$. Then, let us consider two reference tags placed in the positions $\left[x_{\text {tag }, A}, y_{\text {tag }, A}, z_{\text {tag }, A}\right]=[2,2,3] \mathrm{m} \quad$ and $\left[x_{\text {tag }, B}, y_{\text {tag }, B}, z_{\text {tag }, B}\right]=[6.5,2,3] \mathrm{m}$ (red markers in Fig. 2). The phase samples acquired by the RFID reader are shown in Fig. 3, where a standard deviation of $\sigma_{\varphi}=0.1 \mathrm{rad}$ was considered. $N_{r}=2000$ readings were available for each tag along the mobile node trajectory. A window with 100 phase samples is selected and employed as input parameter of the novel phase-based localization method, together with the data of acceleration and angular speed. Thus, they are combined through the Sensor Fusion algorithm. As soon as a new phase sample is available, during the mobile node motion, the localization method procedure is repeated according to a sliding window approach. The trajectory estimated using the novel localization method is represented by the dashed curve in Fig. 2. Instead, the dash-dotted curve represents the trajectory estimated by integrating the data from the inertial platform according to a dead-reckoning approach.

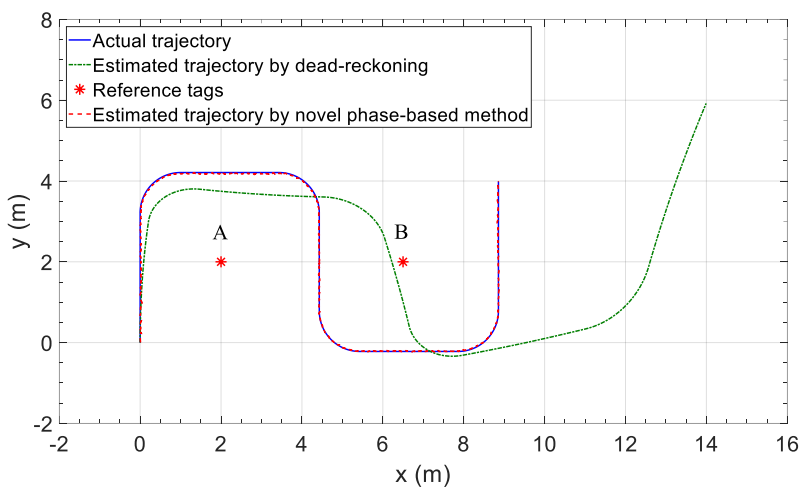

Fig. 2. Simulated "S-shaped" trajectory (solid line) run by the mobile node and estimated trajectories by employing a dead-reckoning approach (dash-dotted line) and the novel UHF-RFID phase-based method (dashed line) with two reference tags placed at $\left[x_{\text {tag }, A}, y_{\text {tag }, A}, z_{\text {tag }, A}\right]=[2,2,3] \mathrm{m}$ and $\left[x_{\text {tag }, B}, y_{\text {tag }, B}, z_{\text {tag }, B}\right]=[6.5,2,3] \mathrm{m}$ (star markers). The average speed of the mobile node is $1 \mathrm{~m} / \mathrm{s}$.

It is apparent that, the new localization method allows to reduce the positioning error with respect to the case in which only the data measured by the kinematic sensors are employed. This effect is clearly visible in Fig. 4 which represents the error on the estimated $x$-coordinate $\varepsilon_{x}$ (Fig. 
4a), on the estimated $y$-coordinate $\varepsilon_{y}$ (Fig. 4b) and the Euclidean distance error between the estimated and the actual trajectories (Fig. 4c), with respect to the distance travelled by the mobile node along the path. By using the IMU data

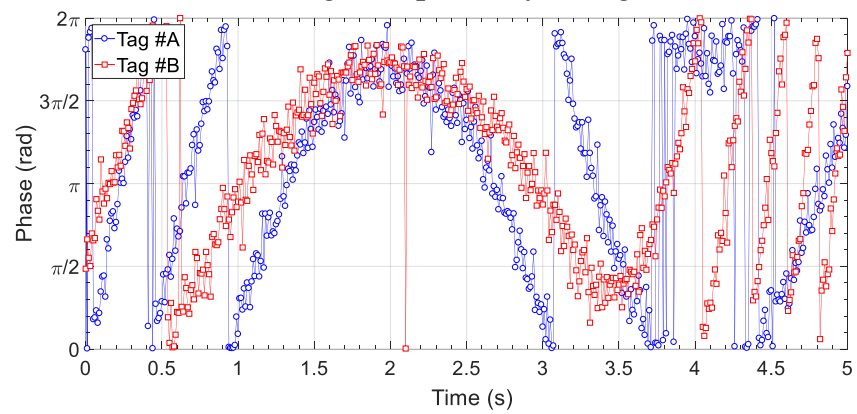

(acceleration and angular velocity) through a dead-reckoning approach, the Euclidean distance error reaches a value of $6 \mathrm{~m}$ (dash-dotted curve) at the end of the path. On the contrary, such an error never exceeds $20 \mathrm{~cm}$ along the mobile node path when using the novel phase-based localization method (dashed curve).

Fig. 3. Phase samples of the backscattered signal of the reference tags placed at $\left[x_{\text {tag }, A}, y_{\text {tag }, A}, z_{\text {tag }, A}\right]=[2,2,3] \mathrm{m} \quad$ (circle markers) and $\left[x_{\text {tag }, B}, y_{\text {tag }, B}, z_{\text {tag }, B}\right]=[6.5,2,3] \mathrm{m}$ (square markers), when the mobile node follows the "S-shaped" trajectory of Fig. 2.

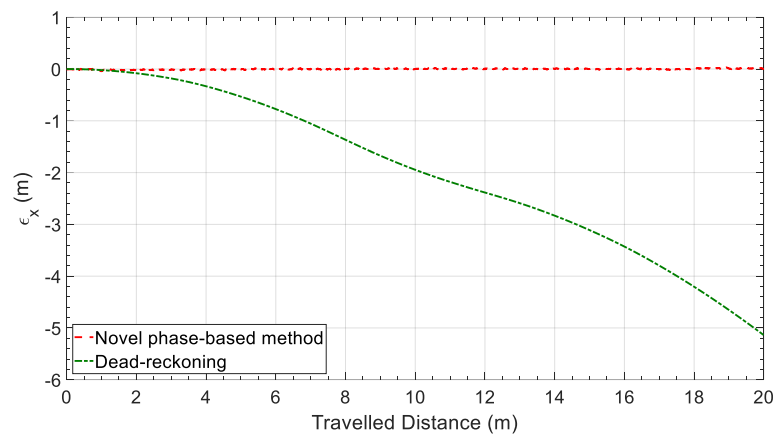

(a)

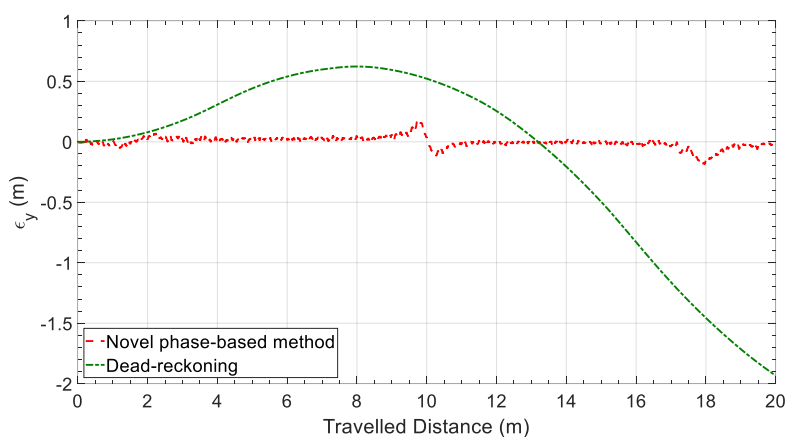

(b)

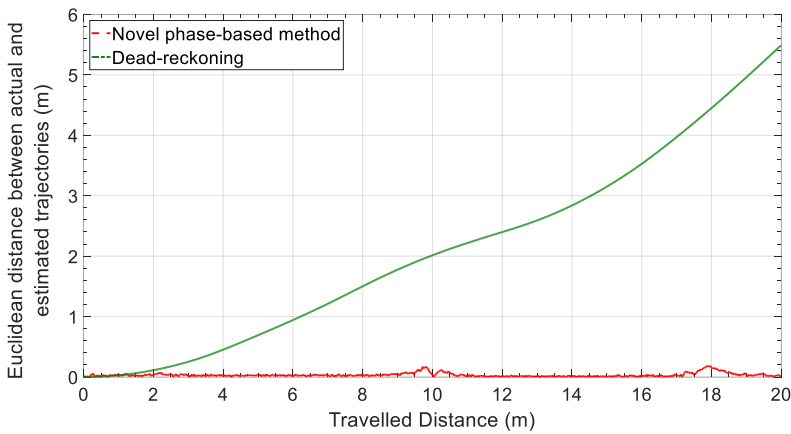

(c)

Fig. 4. Localization error between the actual position of the mobile node (S-shape trajectory of Fig. 2) and the estimated position by employing a dead-reckoning approach (dash-dotted line) or the novel UHF-RFID phasebased method (dashed line) with the two reference tags \#A and \#B. (a) Error on the $x$-coordinate, (b) error on the $y$-coordinate and (c) Euclidean distance error.

As further example, we consider a Lissajous-figure trajectory of the mobile node (Fig. 5-Fig. 6) to evaluate the effect of the number of reference tags identified along the path. 


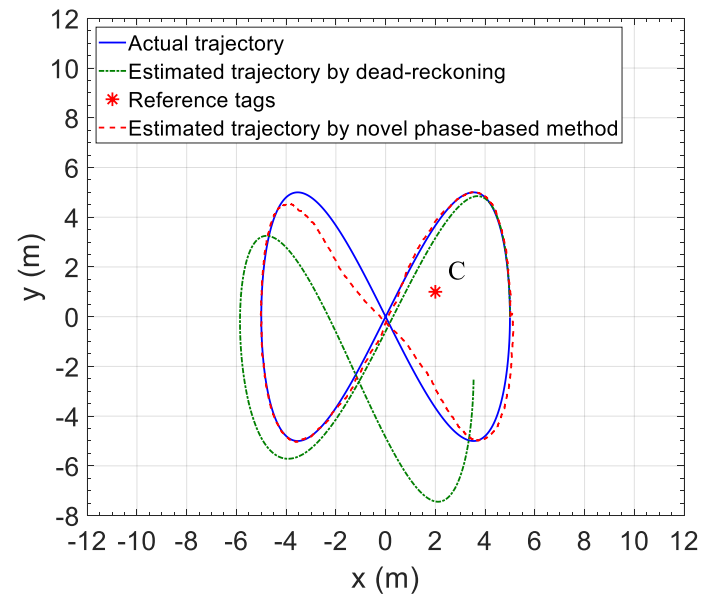

Fig. 5. Simulated "Lissajous-figure" trajectory (solid line) run by the mobile node and estimated trajectories by employing a dead-reckoning approach (dash-dotted line) and the novel UHF-RFID phase-based method (dashed line) with one reference tag placed at $\left[x_{\text {tag, },}, y_{\text {tag }, C}, z_{\text {tag }, C}\right]=[2,1,3] \mathrm{m}$ (star markers). The average speed of the mobile node is $1 \mathrm{~m} / \mathrm{s}$.

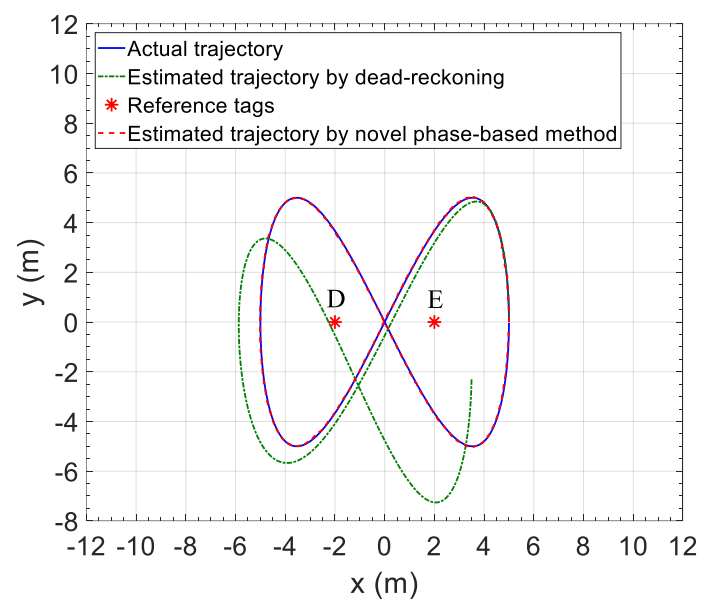

Fig. 6. Simulated "Lissajous-figure" trajectory (solid line) run by the mobile node and estimated trajectories by employing a dead-reckoning approach (dash-dotted line) and the novel UHF-RFID phase-based method (dashed line) with two reference tags placed at $\left[x_{t a g, D}, y_{t a g, D}, z_{t a g, D}\right]=[-2,0,3] \mathrm{m} \quad$ and $\quad\left[x_{t a g, E}, y_{t a g, E}, z_{t a g, E}\right]=[2,0,3] \mathrm{m}$ (star markers). The average speed of the mobile node is $1 \mathrm{~m} / \mathrm{s}$.

The case of a single reference tag placed at $\left[x_{\text {tag }, C}, y_{\text {tag }, C}, z_{\text {tag }, C}\right]=[2,1,3] \mathrm{m}$ (Fig. 5), is compared with the case of two reference tags placed at $\left[x_{\text {tag }, D}, y_{\text {tag }, D}, z_{\text {tag }, D}\right]=[-2,0,3] \mathrm{m} \quad$ and $\left[x_{\text {tag }, E}, y_{\text {tag, },}, z_{\text {tag }, E}\right]=[2,0,3] \mathrm{m}$ (Fig. 6). A window of 100 phase samples is selected and employed as input parameter of the novel phase-based localization method. The average mobile node speed is equal to $2.3 \mathrm{~m} / \mathrm{s}$. Other system parameters are as follow: $a_{x} \in[-0.49,0.49] \mathrm{m} / \mathrm{s}^{2}$, $a_{y} \in[-1.97,1.97] \mathrm{m} / \mathrm{s}^{2}, a_{z}=0, \omega \in[-1.83,1.83] \mathrm{rad} / \mathrm{s}$.

For the case of the reference tag \#C, the estimated trajectory is represented in Fig. 5 by employing the novel localization method (dashed line) and the dead-reckoning approach with IMU data (dash-dotted line). The corresponding localization error is depicted in Fig. 7. The novel method shows improved positioning performance which can be further enhanced by adopting two reference tags, namely tag \#D and \#E, as shown in Fig. 8. In the latter case, the localization error decreases and it is lower than $20 \mathrm{~cm}$ along the considered mobile node path.

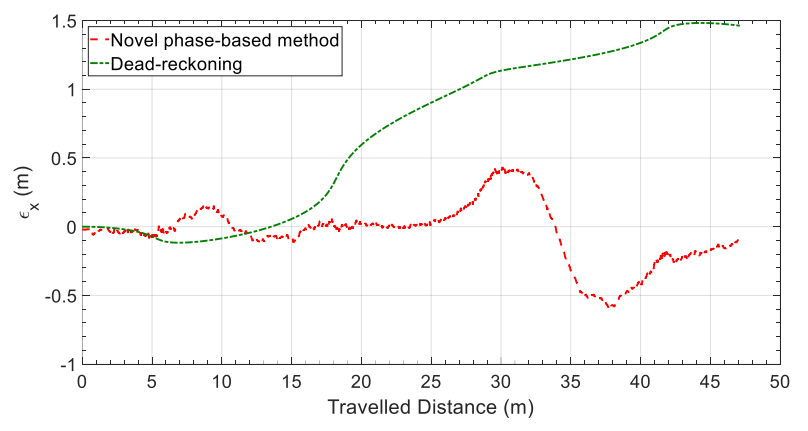

(a)

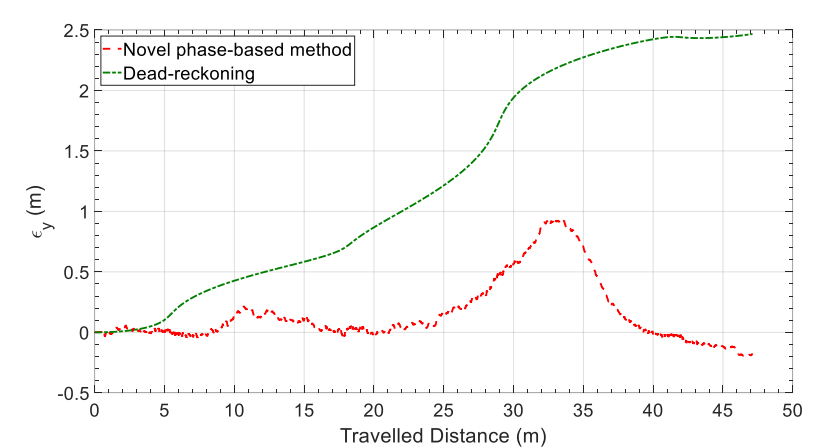

(b)

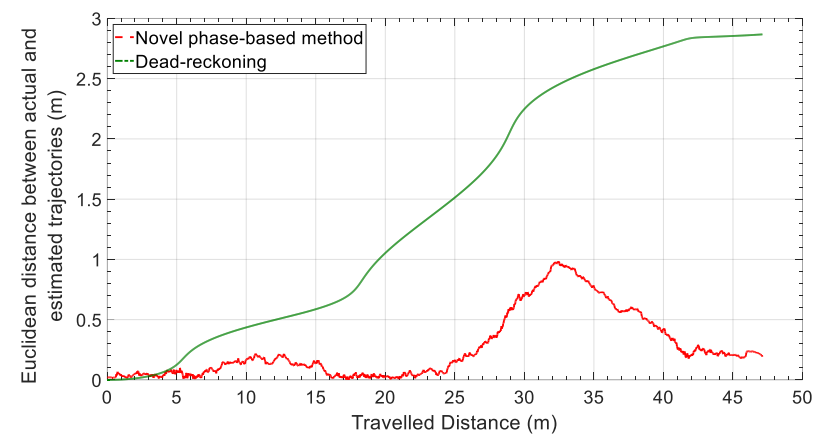

(c)

Fig. 7. Localization error between the actual position of the mobile node (Lissajous-figure trajectory of Fig. 5) and the estimated position by employing a dead-reckoning approach (dash-dotted line) or the novel UHFRFID phase-based method (dashed line) with the reference tag \#C. (a) Error on the $x$-coordinate, (b) error on the $y$-coordinate and (c) Euclidean distance error.

\section{CONCLUSION}

This paper presented a novel phase-based method for mobile node localization through a UHF-RFID system and kinematic sensors. The method exploited the mobile node motion with respect to static reference tags, to collect several phase data, by forming a synthetic array. This allowed to reduce the number of required reference tags in the scenario. The collected phase samples were combined with data acquired by low-cost kinematic sensors through a Sensor Fusion approach. The method can be employed in both indoor and outdoor scenarios and independently on the reference tag positions. Thanks to the UHF-RFID technology, the proposed system allows to reduce the typical drift of dead-reckoning algorithms, with a low-cost and easy deployable solution. 
The implementation of the technological demonstrator of the system is under developed. The result of the experimental analysis will be show at the conference.

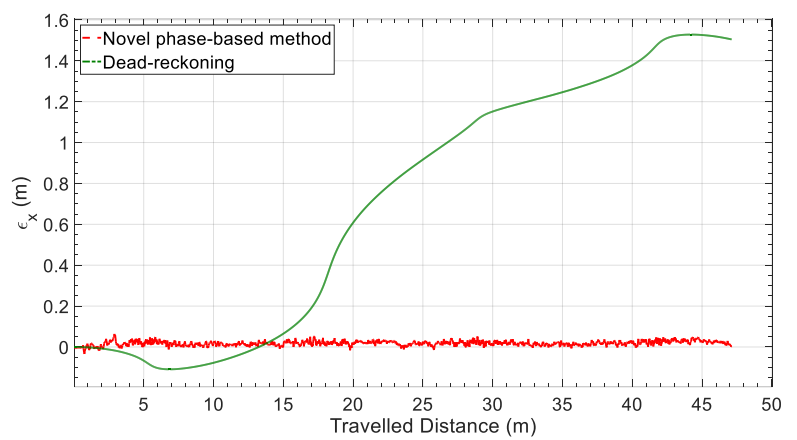

(a)

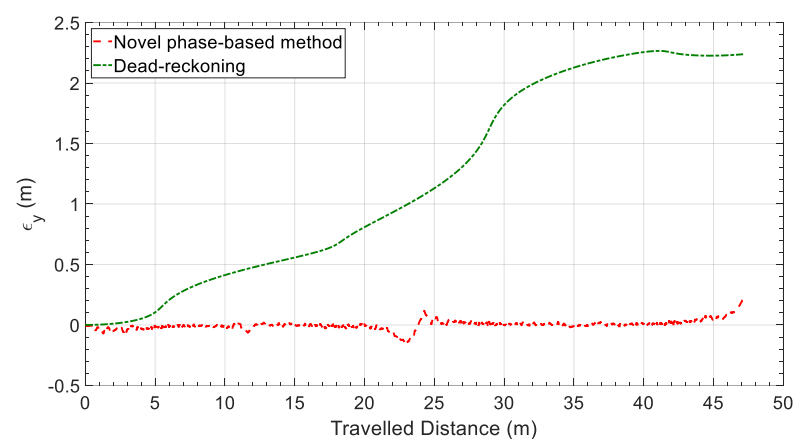

(b)

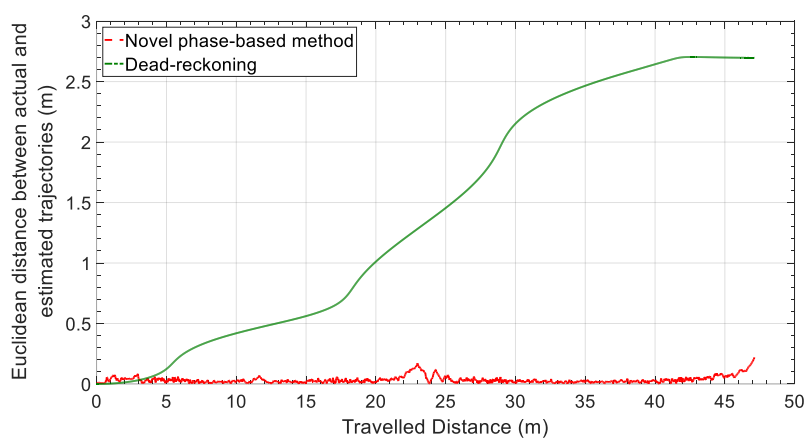

(c)

Fig. 8. Localization error between the actual position of the mobile node (Lissajous-figure trajectory of Fig. 6) and the estimated position by employing a dead-reckoning approach (dash-dotted line) or the novel UHFRFID phase-based method (dashed line) with the two reference tags \#E and \#D. (a) Error on the $x$-coordinate, (b) error on the $y$-coordinate and (c) Euclidean distance error

\section{ACKNOWLEDGMENT}

This work was supported by University of Pisa within the Project AURORA of the "Bando Dimostratori Tecnologici" (D.R 1528, September 13, 2018).

\section{REFERENCES}

[1] C. Laoudias, A. Moreira, S. Kim, S. Lee, L. Wirola, and C. Fischione, "A Survey of Enabling Technologies for Network Localization, Tracking, and Navigation," IEEE Communications Surveys \& Tutorials, vol. 20, no. 4, pp. 3607-3644, Fourthquarter 2018.

[2] H. Liu, H. Darabi, P. Banerjee, and J. Liu, "Survey of wireless indoor positioning techniques and systems," IEEE Transactions on Systems,
Man, and Cybernetics, Part C (Applications and Reviews), vol. 37, no. 6, pp. 1067-1080, Nov. 2007.

[3] N. K. Boggarpu and R. C. Kavanagh, "New learning algorithm for highquality velocity measurement and control when using low-cost optical encoders," IEEE Transactions on Instrumentation and Measurement, vol. 59, no. 3, pp. 565-574, Mar. 2010.

[4] Xian'en Liu, Bojun Ma, Ningning Qi, Zhenxin Zhang, and Dongchun Ren, "A sonar data based particle filtering localization method for mobile robot," 2008 7th World Congress on Intelligent Control and Automation, pp. 3920-3924, Jun. 2008

[5] M. A. H. Ali and M. Mailah, "Path planning and control of mobile robot in road environments using sensor fusion and active force control," IEEE Transactions on Vehicular Technology, vol. 68, no. 3, pp. 21762195, Mar. 2019.

[6] N. V. Jankovic', S. V. C' iric', and N. S. Jovic 'ic', "System for indoor localization of mobile robots by using machine vision," $201523^{\text {rd }}$ Telecommunications Forum Telfor (TELFOR), pp.619-622, Nov. 2015.

[7] M. Bouet and A. L. dos Santos, "Rfid tags: Positioning principles and localization techniques," 2008 1st IFIP Wireless Days, pp. 1-5, Nov. 2008.

[8] T. Sanpechuda and L. Kovavisaruch, "A review of RFID localization: Applications and techniques," 2008 5th International Conference on Electrical Engineering/Electronics, Computer, Telecommunications and Information Technology, Krabi, pp. 769-772, 2008.

[9] Park Ki-Cheol, Park San-Heon, Ko Won-Jun, “Apparatus and method of recognizing position and direction of mobile robot", US2003236590 (A1) US patent, 2003.

[10] H. Ma and K. Wang, "Fusion of RSS and Phase Shift Using the Kalman Filter for RFID Tracking," IEEE Sensors Journal, vol. 17, no. 11, pp. 3551-3558, Jun. 2017.

[11] L. Jing and P. Yang, "A Localization Algorithm for Mobile Robots in RFID System”, 2007 International Conference on Wireless Communications, Networking and Mobile Computing, Shanghai, pp. 2109-2112, 2007.

[12] L. Yang, J. Cao, W. Zhu and S. Tang, "Accurate and Efficient Object Tracking Based on Passive RFID," IEEE Transactions on Mobile Computing, vol. 14, no. 11, pp. 2188-2200, Nov. 2015.

[13] S. S. Saab and Z. S. Nakad, "A Standalone RFID Indoor Positioning System Using Passive Tags," IEEE Transactions on Industrial Electronics, vol. 58, no. 5, pp. 1961-1970, May 2011.

[14] S. Park and H. Lee, "Self-Recognition of Vehicle Position Using UHF Passive RFID Tags," IEEE Transactions on Industrial Electronics, vol. 60, no. 1, pp. 226-234, Jan. 2013.

[15] P. V. Nikitin, R. Martinez, S. Ramamurthy, H. Leland, G. Spiess, and K. V. S. Rao, "Phase based spatial identification of UHF RFID tags," 2010 IEEE International Conference on RFID (IEEE RFID 2010), Orlando, FL, pp. 102-109, 2010

[16] M. B. Akbar, D. G. Taylor, and G. D. Durgin, “Amplitude and phase difference estimation bounds for multisensor based tracking of RFID Tags," 2015 IEEE International Conference on RFID (RFID), San Diego, CA, pp. 105-112, 2015.

[17] S. Sarkka, V. V. Viikari, M. Huusko, and K. Jaakkola, "Phase-based uhf rfid tracking with nonlinear kalman filtering and smoothing," IEEE Sensors Journal, vol. 12, no. 5, pp. 904-910, May 2012.

[18] Q. Yang, D. G. Taylor, M. B. Akbar, and G. D. Durgin, "Analysis of Kalman Filter-Based Localization for HIMR RFID Systems," IEEE Journal of Radio Frequency Identification, Jun. 2019.

[19] E. Di Giampaolo and F. Martinelli, "Mobile Robot Localization Using the Phase of Passive UHF RFID Signals," IEEE Transactions on Industrial Electronics, vol. 61, no. 1, pp. 365-376, Jan. 2014.

[20] F. Martinelli, "A robot localization system combining rssi and phase shift in uhf-rfid signals," IEEE Transactions on Control Systems Technology, vol. 23, no. 5, pp. 1782-1796, Sep. 2015.

[21] A. Buffi, P. Nepa, A. Motroni, and B. Tellini, Patent Application, 2019. 